Pacific Journal of Mathematics

WHEN ARE WITT RINGS GROUP RINGS 


\title{
WHEN ARE WITT RINGS GROUP RINGS?
}

\section{ROGER WARE}

\begin{abstract}
It has been shown that if $C$ is a commutative connected semi-local ring with involution $J$ then the Witt ring, $W(C, J)$, of hermitian forms over $C$ is a factor ring of an integral group ring $Z[G]$, with $G$ a group of exponent two. The purpose of this note is to characterize those pairs $(C, J)$ whose Witt rings are actually isomorphic to integral group rings (Theorem 1 ).
\end{abstract}

I would like to express my thanks to Alex Rosenberg and Manfred Knebusch for several helpful suggestions.

This paper is in part motivated by the result of Elman and Lam which states that if $F$ is a superpythagorean field [3, Th. 4.3, Def. 4.4] then the Witt ring, $W(F)$, of $F$ is isomorphic to a group ring $Z[H]$, where $H$ can be taken to be any subgroup of $F^{*} / F^{* 2}$ of index two, not containing the square class of -1 [3, Th. 5.13 (8)]. In Theorem 1 a different proof of the Elman-Lam result is given and it is shown that the converse is also true. In order to extend the notion of superpythagorean to semi-local rings, we employ the concept of signature as defined in [6].

In what follows $C$ will always be a commutative connected (= no idempotents other than 0 and 1) semi-local ring with involution $J$ and $A$ will be the fixed ring of $J$. We allow the possibility that $J$ is the identity. The groups of units of $C$ and $A$ are denoted by $C^{*}$ and $A^{*}$ respectively, and $N: C^{*} \rightarrow A^{*}$ is the homomorphism given by $N(c)=c J(c)$. We denote by $W(C, J)$ the Witt ring of hermitian spaces over $C$ with respect to the involution $J$, as defined in [5]. The ring theoretic operations of $W(C, J)$ are induced by the orthogonal direct sum and tensor product of spaces respectively. For $a$ in $A^{*}$ we let $\langle a\rangle$ denote the class in $W(C, J)$ of the rank one hermitian space $C$ with form $\left(c_{1}, c_{2}\right) \rightarrow c_{1} J\left(c_{2}\right) a$ and $[a]$ the image of $a$ in the group $A^{*} / N C^{*}$. Then $\langle a\rangle=\langle b\rangle$ in $W(C, J)$ if and only if $[a]=[b]$ in $A^{*} / N C^{*}$ and $\langle a\rangle\langle b\rangle=\langle a b\rangle$. Hence the assignment $[a] \rightarrow\langle a\rangle$ induces a ring homomorphism $\psi: Z\left[A^{*} / N C^{*}\right] \rightarrow W(C, J)$. By [5, Th. 1.16], the mapping $\psi$ is surjective.

A signature $\sigma$ of $(C, J)$ is a group homomorphism $\sigma: A^{*} \rightarrow\{ \pm 1\}$ with the property that $\sigma\left(N C^{*}\right)=1$ and if $\sigma: Z\left[A^{*} / N C^{*}\right] \rightarrow Z$ also denotes the induced ring homomorphism then $\sigma(\operatorname{Ker} \psi)=0$. As remarked in [6], the signatures of $(C, J)$ correspond bijectively with the ring homomorphisms from $W(C, J)$ to $Z$. By [5, Example 3.11] the latter set is in bijective correspondence with the set of non-maximal prime ideals of $W(C, J)$. If $J$ is the identity and $C=A$ is a field 
then the signatures of $C$ correspond to the (total) orderings on $C$ (cf. [6, Remark 1.7 (ii)]).

Since the kernel of the natural map $\psi: Z\left[A^{*} / N C^{*}\right] \rightarrow W(C, J)$ contains the element [1] $+[-1]$, [5, Cor. 1.17], it follows that any signature $\sigma$ of $(C, J)$ has the property that $\sigma(-1)=-1$. Suppose, in addition, $(C, J)$ has the following property

(*) $C$ has no maximal ideal $M$ with $J(M)=M$ such that either $C / M=\boldsymbol{F}_{2}$ or $C / M=\boldsymbol{F}_{4}$ and $A / M \cap A=\boldsymbol{F}_{2}\left(\boldsymbol{F}_{n}=\right.$ finite field with $n$ elements).

Then, by [6, Prop. 1.4], a homomorphism $\sigma: A^{*} \rightarrow\{ \pm 1\}$ with $\sigma\left(N C^{*}\right)=1$ and $\sigma(-1)=-1$ is a signature if and only if $\sigma(a)=1$ implies $\sigma\left(N\left(c_{1}\right)+a N\left(c_{2}\right)\right)=1$ for any $c_{1}, c_{2}$ in $C$ with $N\left(c_{1}\right)+a N\left(c_{2}\right)$ in $A^{*}$.

The main result is the following.

Theorem 1. Assume $C$ has property $\left(^{*}\right)$ and -1 is not in $N C^{*}$. Then the following statements are equivalent:

(i) For any $a$ in $A^{*}$ with $a \notin-N C^{*}$ we have $(N C+a N C) \cap A^{*}=N C^{*} \cup a N C^{*}$.

(ii) If $\sigma: A^{*} \rightarrow\{ \pm 1\}$ is a homomorphism such that $\sigma\left(N C^{*}\right)=1$ and $\sigma(-1)=-1$ then $\sigma$ is a signature of $(C, J)$.

(iii) If $E$ is a finite subgroup of $A^{*} / N C^{*}$ not containing the norm class [-1] then there exists a signature $\sigma$ of $(C, J)$ such that $\sigma(E)=1$.

(iv) If $H$ is any subgroup of $A^{*} / N C^{*}$ not containing [-1] then there exists a signature $\sigma$ such that $\sigma(H)=1$.

(v) The kernel of the mapping $\psi: Z\left[A^{*} / N C^{*}\right] \rightarrow W(C, J)$ is the ideal generated by $[1]+[-1]$.

(vi) $W(C, J) \cong Z[H]$ where $H$ is a subgroup of index two in $A^{*} / N C^{*}$. The group $H$ can be taken to be any subgroup of index two not containing [-1].

(vii) $W(C, J) \cong Z[H]$ for some group $H$ of exponent two.

Proof. (i) $\Rightarrow$ (ii) As mentioned above it is enongh to show that if $a$ is a unit of $A$ with $\sigma(a)=1$ and $c_{1}, c_{2}$ are elements of $C$ such that $b=N\left(c_{1}\right)+a N\left(c_{2}\right)$ is also a unit then $\sigma(b)=1$. Since $\sigma(a)=1$ it follows that $a \notin-N C^{*}$. Hence by (i), $b$ lies in $N C^{*} \cup a N C^{*}$ so that $\sigma(b)=1$, as desired.

(ii) $\Rightarrow$ (iii) is clear.

(iii) $\Rightarrow$ (iv). Let $\operatorname{Sign}(C, J)$ denote the set of signatures of $(C, J)$ and for $a$ in $A^{*}$, let $V(a)=\{\sigma$ in $\operatorname{Sign}(C, J) \mid \sigma(a)=1\}$. The sets $V(a)$, $a$ in $A^{*}$, can be taken as a subbase for a topology on $\operatorname{Sign}(C, J)$ which makes $\operatorname{Sign}(C, J)$ a compact Hausdorff space and each $V(a)$ a closed set [6, Lemma 2.3, Th. 2.18, Lemma 3.3 (i)]. Now let $H$ be a subgroup of $A^{*} / N C^{*}$ with $[-1] \notin H$ and choose $\left\{a_{i}\right\}_{i \in I} \subset A^{*}$ such that $H=\left\{\left[a_{i}\right]\right\}_{i \in I}$. 
For any finite subset $I_{0} \subset I$, the group $H_{0}$ generated by $\left\{\left[a_{i}\right]\right\}_{i \in I_{0}}$ is finite and $[-1] \notin H_{0}$. Hence, by (iii), there exists a signature $\sigma$ such that $\sigma\left(H_{0}\right)=1$, i.e., $\bigcap_{i \in I_{0}} V\left(a_{i}\right) \neq \varnothing$. Thus $\left\{V\left(a_{i}\right)\right\}_{i \in I}$ is a family of closed sets with the finite intersection property. Since $\operatorname{Sign}(C, J)$ is compact it follows that $\bigcap_{i \in I} V\left(a_{i}\right) \neq \varnothing$, i.e., there exists a signature $\sigma$ with $\sigma(H)=1$.

(iv) $\Rightarrow(v)$. Let $G=A^{*} / N C^{*}$ and let $L$ be the ideal of $Z[G]$ generated by [1] $+[-1]$. Now, minimal prime ideals of $Z[G]$ correspond bijectively with group homomorphisms $G \rightarrow\{ \pm 1\}$ and under this correspondence, prime ideals containing $L$ correspond to homomorphisms sending [-1] to -1 [5, Lemma 3.1]. By (iv), the latter set coincides with the set of signatures of $(C, J)$. Thus if $K$ is the kernel of the mapping $\psi$, then $L \subset K$ and if $P$ is a minimal prime ideal of $Z[G]$ with $L \subset P$ then by definition of signature, we must also have $K \subset P$. Thus to prove (v) it is enough to show that $L$ is the intersection of all such prime ideals. This is done by showing that $Z[G] / L \cong Z[H]$ where $H$ is any subgroup of index two in $G$ with $[-1] \notin H$. Statement (v) then follows because $Z[H]$ has no nonzero nilpotent elements. Note that this will also prove the implication $(\mathrm{v}) \Rightarrow(\mathrm{vi})$.

Thus let $H$ be a subgroup of index two in $G$ with [-1] $\notin H$. Let $S=\{[1],-[-1]\}$ and $G^{\prime}=H \times S$. Then $Z[G]=Z\left[G^{\prime}\right]$ and $L$ is the ideal generated by all elements of the form $1-s, s \in S$. Hence $\boldsymbol{Z}[G] / L=\boldsymbol{Z}\left[G^{\prime}\right] / L \cong \boldsymbol{Z}\left[G^{\prime} / S\right] \cong \boldsymbol{Z}[H]$.

(v) $\Rightarrow$ (vi) is contained in the above argument.

(vi) $\Rightarrow$ (vii) is trivial.

(vii) $\Rightarrow$ (i). Let $H$ be a group of exponent two and $f: W(C, J) \rightarrow Z[H]$ an isomorphism. Since for any $a$ in $A^{*},\langle a\rangle^{2}=1$ in $W(C, J)$ it follows that $(f(\langle a\rangle))^{2}=1$ in $Z[H]$ so by [5, Th. 3.23], $f(\langle a\rangle)= \pm h$ for some $h$ in $H$. Now suppose $a$ is a unit in $A$ with $a \notin N C^{*}$ and $c_{1}, c_{2}$ are elements of $C$ such that $b=N\left(c_{1}\right)+a N\left(c_{2}\right)$ is a unit in $A$. Then by [5, Th. 1.16 (iii) and Lemma 1.19] $(1+\langle a\rangle)(1-\langle b\rangle)=0$ in $W(C, J)$. Hence

$$
1=-f(\langle a\rangle)+f(\langle b\rangle)+f(\langle a b\rangle)
$$

in $Z[H]$. Thus either $f(\langle a\rangle)=-1$, or $f(\langle b\rangle)=1$, or $f(\langle a b\rangle)=1$. Since $f$ is an isomorphism, $f(\langle a\rangle)=-1$ implies $\langle a\rangle=\langle-1\rangle$ in $W(C, J)$ which implies $a \in-N C^{*}$, contrary to assumption. If $f(\langle b\rangle)=1$ then $\langle b\rangle=1$ in $W(C, J)$, i.e., $b=N\left(c_{1}\right)+a N\left(c_{2}\right) \in N C^{*}$, so we are done in this case. If $f(\langle a b\rangle)=1$ then $f(\langle a\rangle)=f(\langle b\rangle)$ so $\langle a\rangle=\langle b\rangle$, i.e., $b \in a N C^{*}$, completing the proof.

REMARKs. (i ) In [3], Elman and Lam studied formally real (= ordered) fields satisfying condition (iii) of Theorem 1. There, they 
proved a structure theorem, [3, Th. 5.13], for the Witt ring and algebraic $k$-groups of such fields which contains the statement that the Witt ring is an integral group ring. They also proved several equivalent conditions characterizing these fields which include the equivalence of (iii) and (iv) [3, Ths. 4.3, 4.7]. In fact, the foregoing proof of (iii) $\Rightarrow$ (iv) is the same as the proof $\mathrm{S} 1 \Rightarrow \mathrm{S} 2$ in Theorem 4.3.

(ii) Diller and Dress [2] observed the equivalence of conditions (i) and (ii) when $J=$ Identity and $C=A$ is a field and showed that these are equivalent to the following:

For any $a$ in $A^{*}$ with $a \notin-A^{* 2}$ the field $A(\sqrt{a})$ is pythagorean, i.e., sums of squares are squares [2, Satz 4].

Following Elman-Lam [3, Def. 4.4], we call $(C, J)$ superpythagorean if $(C, J)$ has property $(*)$ and satisfies the conditions of Theorem 1 .

CoRollary 2. If $(C, J)$ is superpythagorean then every unit of $A$ which is a sum of norms is itself a norm.

Proof. This follows from condition (i) with $a=1$. (See also [6, Prop. 3.13].)

Corollary 3. (cf. [3, Cor. 4.5]). Assume $A^{*} / N C^{*}$ is a finite group of order $2^{n}, n \geqq 1$. Then $(C, J)$ is superpythagorean if and only if $C$ has exactly $2^{n-1}$ distinct signatures.

Proof. Apply condition (ii) of the theorem together with the fact that there are exactly $2^{n-1}$ homomorphisms $A^{*} / N C^{*} \rightarrow\{ \pm 1\}$ sending $[-1]$ to -1 .

REMARK. In contrast to the Witt ring, the Witt-Grothendieck ring $K(C, J)$ of isometry classes of nondegenerate hermitian spaces over $C$ is seldom an integral group ring. In fact, if $-1 \notin N C^{*}$, it is not difficult to show that the following statements are equivalent:

(a) $K(C, J)$ is the integral group ring of some group,

(b) $K(C, J) \cong Z\left[A^{*} / N C^{*}\right]$,

(c) $A^{*} / N C^{*}$ is cyclic of order two,

(d) $W(C, J) \cong \boldsymbol{Z}$,

(e) Ker $\psi$ is additively generated by [1] $+[-1]$.

For the remainder of the paper we assume that $J$ is the identity (so $C=A$ and $N C^{*}=A^{* 2}$ ).

Proposition 4. Let $A$ be a local ring with maximal ideal $M$ and residue class field $k=A / M$. Assume $1+M \subset A^{* 2}$ (this happens, for example, if $A$ is henselian [1, Ex. 3, p. 126]). Then

(i) $A$ is superpythagorean if and only if $k$ is a superpythagorean field. 
(ii) If, in addition, $A$ is a valuation ring with field of fractions $F$ then $A$ is superpythagorean if and only if $F$ is a superpythagorean field.

Proof. (i ) By [4, Satz 7.1.1, N.B. 7.1.3] there is an isomorphism of Witt rings $W(A) \cong W(k)$ and hence $A$ is superpythagorean if and only if $k$ is.

(ii) Let $A$ be a valuation ring with field of fractions $F$ and assume $A$ is superpythagorean. Since $F$ is a field, in order to show a function $\sigma: F^{*} \rightarrow\{ \pm 1\}$ with $\sigma\left(F^{* 2}\right)=1$ and $\sigma(-1)=-1$ is a signature it is enough to show that $\sigma(a)=1$ implies $\sigma(1+a)=1$. Thus suppose $a$ is an element of $F^{*}$ with $\sigma(a)=1$ and let $\bar{\sigma}=\sigma \mid A^{*}$. Then $\bar{\sigma}\left(A^{* 2}\right)=1$ and $\bar{\sigma}(-1)=-1$ so $\bar{\sigma}$ is a signature of $A$. Since $A$ is a valuation ring of $F$, for any $a$ in $F$, either $a$ is a unit in $A$, or $a \in M$, or $a^{-1} \in M$. If $a$ is a unit in $A$ then $1+a$ is also a unit (if $1+a \in M$ then $1+m=-a$ for some $m \in M$ and since $1+M \subset A^{* 2}$ this means $1=\sigma(1+m)=\sigma(-a)=-\sigma(a)=-1$, impossible). Since $\bar{\sigma}$ is a signature, $\sigma(1+a)=\bar{\sigma}(1+a)=1$. If $a \in M$ then $1+a \in A^{* 2}$ so $\sigma(1+a)=1$ and if $a^{-1} \in M$ then $\sigma\left(1+a^{-1}\right)=1$ and

$\sigma(1+a)=\sigma\left(a\left(a^{-1}+1\right)\right)=\sigma(a)\left(a^{-1}+1\right)=1$, showing that $F$ is superpythagorean.

Conversely, suppose $F$ is superpythagorean. Since $A$ is integrally closed in $F$, the inclusion $A^{*} \rightarrow F^{*}$ induces an inclusion $A^{*} / A^{* 2} \rightarrow F^{*} / F^{* 2}$. Since both are vector spaces over $\boldsymbol{F}_{2}$ any homomorphims $\sigma: A^{*} / A^{* 2} \rightarrow\{ \pm 1\}$ extends to a homomorphism $\hat{\sigma}: F^{*} / F^{* 2} \rightarrow\{ \pm 1\}$. If $\hat{\sigma}$ is a signature of $F$ then $\sigma$ is a signature of $A$, completing the proof.

REMARK. The last part of the proof actually shows that if $A \subset B$ are rings with $A^{*} \cap B^{* 2}=A^{* 2}$ then $A$ is superpythagorean if $B$ is.

ExAmples. Assume $k$ is a superpythagorean field. Then

(a) The ring of formal power series in $n$-variables, $k\left[\left[X_{1}, \cdots\right.\right.$, $\left.\left.X_{n}\right]\right]$, is superpythagorean. The ring of dual numbers over $k, k[\varepsilon], \varepsilon^{2}=0$, is superpythagorean.

(b) If $n \geqq 2$ the quotient field $k\left(\left(X_{1}, \cdots, X_{n}\right)\right)$ of $k\left[\left[X_{1}, \cdots, X_{n}\right]\right]$ is not pythagorean (hence cannot be superpythagorean). However,

(c) [3, Cor. 4.6]. For any $n \geqq 1$ the field $\left.k\left(\left(X_{1}\right)\right) \cdots\left(X_{n}\right)\right)$ of iterated Laurent series over $k$ is superpythagorean.

Proof. (a) This is immediate from Proposition 4 (i).

(b) It is not difficult to check that $X^{2}+Y^{2}$ cannot be a square in the field $k((X, Y))$.

(c) Here it is enough to show that $k((X))$ is superpythagorean. However, this follows from (a) and Proposition 4 (ii). 


\section{REFERENCES}

1. N. Bourbaki, Algèbre commutative, Chaps. 3-4, Actualités Sci. Indust., no. 1293, Hermann, Paris, 1967.

2. J. Diller und A. Dress, Zur Galoistheorie pythagoreischer Körper, Arch. Math., 16 (1965), 148-152.

3. R. Elman and T. Y. Lam, Quadratic forms over formally real fields and pythagorean fields, Amer. J. Math., 94 (1972), 1155-1194.

4. M. Knebusch, Grothendieck-und Wittringe von nichtausgearten symmetrischen Bilinear formen, S.-B. Heidelberger Akad. Wiss. Math.-Natur. Kl. 1969, 93-157.

5. M. Knebusch, A. Rosenberg and R. Ware, Structure of Witt rings and quotients of abelian group rings, Amer. J. Math., 94 (1972), 119-155.

6. —_ Signatures on semi-local rings, J. Algebra, 26 (1973), 208-250.

Received July 5, 1972. Partially supported by NSF Grant GP-28915.

NORTHWESTERN UNIVERSITY

Current address: UNIVERSITY OF KANSAS 


\section{PACIFIC JOURNAL OF MATHEMATICS}

\section{EDITORS}

RICHARD ARENS (Managing Editor) University of California

Los Angeles, California 90024

R. A. Beaumon'T

University of Washington Seattle, Washington 98105
J. Dugundj1*

Department of Mathematics University of Southern California Los Angeles, California 90007

D. Gilbarg and J. Milgram Stanford University Stanford, California 94305

\section{ASSOCIATE EDITORS}

E. F. BECKENBACH

B. H. NeUMANN

F. WOLF

K. YOSHIDA

\section{SUPPORTING INSTITUTIONS}

UNIVERSITY OF BRITISH COLUMBIA CALIFORNIA INSTITUTE OF TECHNOLOGY UNIVERSITY OF CALIFORNIA MONTANA STATE UNIVERSITY UNIVERSITY OF NEVADA NEW MEXICO STATE UNIVERSITY OREGON STATE UNIVERSITY UNIVERSITY OF OREGON OSAKA UNIVERSITY

\section{UNIVERSITY OF SOUTHERN CALIFORNIA STANFORD UNIVERSITY UNIVERSITY OF TOKYO UNIVERSITY OF UTAH WASHINGTON STATE UNIVERSITY UNIVERSITY OF WASHINGTON AMERICAN MATHEMATICAL SOCIETY NAVAL WEAPONS CENTER}

* C. R. DePrima California Institute of Technology, Pasadena, CA 91109, will replace J. Dugundji until August 1974. 


\section{Pacific Journal of Mathematics}

\section{Vol. 49, No. $1 \quad$ May, 1973}

A. Bigard, Free lattice-ordered modules ...........................

Richard Bolstein and Warren R. Wogen, Subnormal operators in strictly cyclic

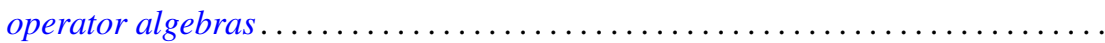

Herbert Busemann and Donald E. Glassco, II, Irreducible sums of simple

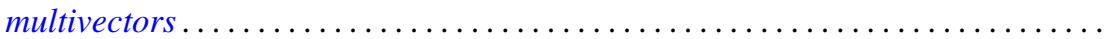

W. Wistar (William) Comfort and Victor Harold Saks, Countably compact groups

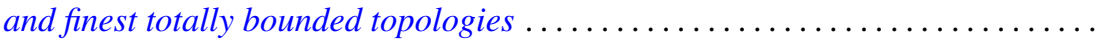

Mary Rodriguez Embry, Maximal invariant subspaces of strictly cyclic operator

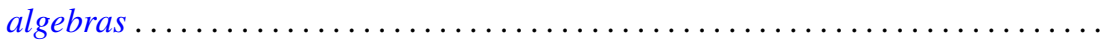

Ralph S. Freese and James Bryant Nation, Congruence lattices of semilattices......

Ervin Fried and George Grätzer, A nonassociative extension of the class of

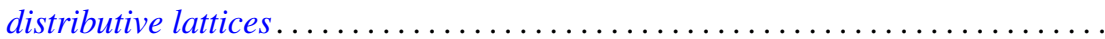

John R. Giles and Donald Otto Koehler, On numerical ranges of elements of locally

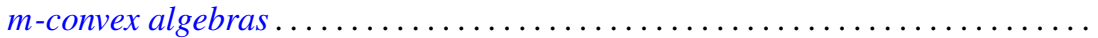

David A. Hill, On dominant and codominant dimension of $\mathrm{QF}-3$ rings ........ John Sollion Hsia and Robert Paul Johnson, Round and Pfister forms over $R(t) \ldots$ I. Martin (Irving) Isaacs, Equally partitioned groups . . . . . . . . . . . . . .

Athanassios G. Kartsatos and Edward Barry Saff, Hyperpolynomial approximation of solutions of nonlinear integro-differential equations.

Shin'ichi Kinoshita, On elementary ideals of $\theta$-curves in the 3-sphere and 2-links in the 4-sphere

Ronald Brian Kirk, Convergence of Baire measures

R. J. Knill, The Seifert and Van Kampen theorem via regular covering spaces ..

Amos A. Kovacs, Homomorphisms of matrix rings into matrix rings ..

Young K. Kwon, HD-minimal but no $H D$-minimal ..........

Makoto Maejima, On the renewal function when some of the mean renewal lifetimes are infinite

Juan José Martínez, Cohomological dimension of discrete modules over profinite groups.

W. K. Nicholson, Semiperfect rings with abelian group of units

Louis Jackson Ratliff, Jr., Three theorems on imbedded prime divisors of principal ideals.

Billy E. Rhoades and Albert Wilansky, Some commutants in $B(c)$ which are almost matrices

John Philip Riley Jr., Cross-sections of decompositions . . .

Keith Duncan Stroyan, A characterization of the Mackey uniformity $m\left(L^{\infty}, L^{1}\right)$ for

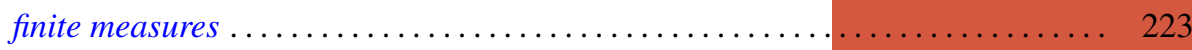

Edward G. Thurber, The Scholz-Brauer problem on addition chains . . . . . . . . . 229

Joze Vrabec, Submanifolds of acyclic 3-manifolds ............

Philip William Walker, Adjoint boundary value problems for compactified singular

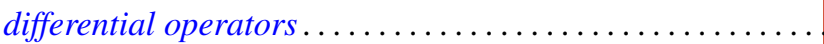

